\title{
Editorial
}

\section{Smart Antennas and Intelligent Sensors Based Systems: Enabling Technologies and Applications}

\author{
Fawad Zaman (iD, ${ }^{1}$ Sungchang Lee ${ }^{(D)},{ }^{2}$ Mohamad K. A. Rahim, ${ }^{3}$ and Sarmadullah Khan ${ }^{4}$ \\ ${ }^{1}$ Department of Electrical and Computer Engineering, COMSATS University Islamabad, Attock Campus, Attock, Pakistan \\ ${ }^{2}$ Korea Aerospace University, Goyang-si, Republic of Korea \\ ${ }^{3}$ Universiti Teknologi Malaysia (UTM), Johor, Malaysia \\ ${ }^{4}$ De Montfort University, Leicester, UK \\ Correspondence should be addressed to Fawad Zaman; fawad@ciit-attock.edu.pk
}

Received 3 June 2019; Accepted 3 June 2019; Published 14 July 2019

Copyright (C) 2019 Fawad Zaman et al. This is an open access article distributed under the Creative Commons Attribution License, which permits unrestricted use, distribution, and reproduction in any medium, provided the original work is properly cited.

The growing communication and computing capabilities in the devices enlarge the connected world and improve the human life comfort level. The evolution of intelligent sensor networks and smart antennas has led to the development of smart devices and systems for real-time monitoring of various environments. The demand of smart antennas and intelligent sensors significantly increases when dealing with multiuser communication system that needs to be adaptive, especially in unknown adverse environment [1-3]. The smart antennas based arrays are capable of steering the main beam in any desired direction while placing nulls in the unwanted directions. Intelligent sensor networks integration with smart antennas will provide algorithms and interesting application to collect various data of environment to make intelligent decisions $[4,5]$.

The aim of this special issue is to provide an inclusive vision on the current research in the area of intelligent sensors and smart antenna based systems for enabling various applications and technologies. We cordially invite some researchers to contribute papers that discuss the issues arising in intelligent sensors and smart antenna based system. Hence, this special issue offers the state-of-the-art research in this field.

The paper titled "Compressive Sensing Based Channel Estimation for Massive MIMO Communication Systems" discusses the advantage of spatial and temporal common sparsity of massive MIMO channels in delay domain, nonorthogonal pilot design, and channel estimation schemes which are proposed under the frame work of structured compressive sensing (SCS) theory that considerably reduces the pilot overheads for massive MIMO FDD systems. Finally, simulations are carried out to verify the performance of the proposed schemes and compared to its conventional counterparts with fewer pilots overhead, the proposed schemes improve the performance of the system.

The paper titled "Generalized Complex Quadrature Spatial Modulation" proposes two generalizations of CQSM, namely, generalized CQSM with unique combinations (GCQSM-UC) and with permuted combinations (GCQSMPC). These two generalizations perform close to CQSM or outperform it, depending on the system parameters. Also, the proposed schemes require much less transmit antennas to achieve the same spectral efficiency of CQSM, for instance, assuming 16-QAM, GCQSM-PC, and GCQSM-UC require 10 and 15 transmit antennas, respectively, to achieve the same spectral of CQSM which is equipped with 32 antennas.

The paper titled "A Compact Ku-Band Active Electronically Steerable Antenna with Low-Cost 3D T/R Module" presents a novel compact Ku-band active electronically steerable antenna array design with a low-cost and integrated T/R 3D module employed for airborne synthetic aperture radar (SAR) systems. The entire system adopts 3D multilayer technology with vertical interconnection to construct the hermetically packaging RF modules. By assembling different multifunctional modules into a whole multilayer board, the $3 \mathrm{D} T / \mathrm{R}$ technique greatly improves the system integration and reduces implementation cost and size.

The paper titled "SUBBASE: An Authentication Scheme for Wireless Sensor Networks Based on User Biometrics" proposes a new method of security for a wireless sensor 
network (WSN). The proposed technique, Secure User Biometric Based Authentication Scheme (SUBBASe), is based on the user biometrics for WSNs. It achieves a higher security level, as well as improved network performance.

The paper titled "Enabling Noninvasive Physical Assault Monitoring in Smart School with Commercial Wi-Fi Devices" presents Wi-Dog, a noninvasive physical assault monitoring scheme that enables privacy-preserving monitoring in ubiquitous circumstances. The key intuition is that WiFi signals are easily distorted by human motions, and motioninduced signals could convey informative characteristics, such as intensity, regularity, and continuity. Specifically, to explicitly reveal the substantive properties of physical assault, the authors propose a set of signal processing methods for informative components extraction by selecting sensitive antenna pairs and subcarriers. Then a novel signal-complexity-based segmentation method is developed as a locationindependent indicator to monitor targeted movement transitions. The Wi-Dog is implemented on commercial Wi-Fi devices and evaluated in real indoor environments.

The paper titled "A Double Adaptive Approach to Tackle Malicious Users in Cognitive Radio Networks" proposes a double adaptive thresholding technique in order to differentiate legitimate users from doubtful and malicious users. Prior to the double adaptive approach, the maximal ratio combining (MRC) scheme is utilized to assign weight to each user such that the legitimate users experience higher weights than the malicious users. Double adaptive threshold is applied to give a fair chance to the doubtful users to ensure their credibility. A doubtful user that fails the double adaptive threshold test is declared as a malicious user. The results of the legitimate users are combined at the fusion center by utilizing Dempster-Shafer (DS) evidence theory. Effectiveness of the proposed scheme is proved through simulations by comparing with the existing schemes.

The paper titled "Smart Real-Time Video Surveillance Platform for Drowsiness Detection Based on Eyelid Closure" proposes drowsiness detection in real-time surveillance videos by determining if a person's eyes are open or closed. As a first step, the face of the subject is detected in the image. In the detected face, the eyes are localized and filtered with an extended Sobel operator to detect the curvature of the eyelids. Once the curves are detected, concavity is used to tell whether the eyelids are closed or open. Consequently, a concave upward curve means the eyelid is closed whereas a concave downwards curve means the eye is open. The proposed method is also implemented on hardware in order to be used in real-time scenarios, such as driver drowsiness detection.

The paper titled "Underwater Wireless Sensor Networks: A Review of Recent Issues and Challenges" conducts a survey of UWSN regarding underwater communication channel, environmental factors, localization, media access control, routing protocols, and effect of packet size on communication. The presently available methodologies are compared and their pros and cons are discussed to highlight new directions of research for further improvement in underwater sensor networks.
The paper titled "An Angle Estimation Method for Monostatic MIMO Radar Based on RCC-FLOM Algorithm" solves the angle estimation problem of coherent sources in the impulse noise background; a conjugate rotation invariant subspace algorithm based on reduced order fractional lower order covariance matrix is proposed. According to the conjugate rotation invariant subspace, the coherent source is decohered. The Monte-Carlo experiments show that the proposed algorithm has the advantages of high estimation probability and low root mean square error in the case of low signal-to-noise ratio, compared with the existing FLOMMUSIC algorithm and FLOM-Unitary ESPRIT algorithm.

The paper titled "A Novel Study to Predict Trends and Policies for Mobile Communication in Multienvironment Regions" explores the usage of mobile communication to analyze the trends; for this, the authors select Khyber Pakhtunkhwa (KPK), Pakistan, a multienvironment based on farmers, students, employees, and businessmen. A field survey is carried out by designing a detailed structural questionnaire for viable collection of data to have superior analysis of different multienvironment based classes. A statistical analysis is performed through hypotheses and chi-square test on a large dataset based on sufficient number of observations collected through primary survey for each class. The survey results are provided in number of graphical and numerical illustrations to predict the mobile usage trends, evidently and effectively, of the people of KPK.

The paper titled "Flexible Queuing Model for Number of Active Users in Cognitive Radio Network Environment" presents a Soft Queuing Model (SQM) for number of active users present in a cognitive radio network (CRN) at some given instant. It starts with the existing cellular network where the upper limit for the number of channels and active users is well defined. The idea is then extended to the complicated scenario of CRNs where the upper limit is not deterministic for both the number of channels and the active users. Accordingly a probabilistic SQM is proposed under the condition that the number of channels and active cognitive users are both random variables. The proposed model will be useful to offer the level of reliability to the clients connected with CRN and hence to offer secure communication even on a cooperative CRN. The proposed model has been verified theoretically and simulations have been carried out in diversified set-ups to evaluate the performance.

The paper titled "Architecture for Collision-Free Communication Using Relaxation Technique" proposes an idea for collision problem in which network relaxation technique is used which is based on fast clique detection. The proposed approach results in high throughput in terms of latency and complexity. Furthermore, the proposed solution is able to solve the collision problem by connecting network optimization for achieving high throughput.

The paper titled "One-to-Many Relationship Based Kullback Leibler Divergence against Malicious Users in Cooperative Spectrum Sensing" proposes a technique based on Kullback Leibler Divergence (KLD) algorithm for mitigating the MUs attack in CSS. The secondary users (SUs) inform FC about the primary user (PU) spectrum availability by sending received energy statistics. Unlike the previous KLD 
algorithm where the individual SU sensing information is utilized for measuring the KLD, in this work MUs are identified and separated based on the individual SU decision and the average sensing statistics received from all other users. The proposed KLD assigns lower weights to the sensing information of MUs, while the normal SUs information receives higher weights. The proposed method has been tested in the presence of always yes, always no, opposite, and random opposite MUs.

The paper titled "Backtracking Search Optimization Paradigm for Pattern Correction of Faulty Antenna Array in Wireless Mobile Communications" presents viable, simple, and accurate stochastic solver based on Backtracking Search Optimization Algorithm (BSA) for the pattern correction of faulty antenna array in mobile communication systems. A fitness function is developed to optimize the weights of the remaining healthy antenna elements in the array. The fitness function consists of two parts; the first part is based on mean square error approach for the reduction of sidelobes level, while, in the second part, steering vectors are used for the repositioning of nulls. Simulation results establish the validity of the BSA from its counterparts based on genetic algorithm and its memetic combination with pattern search technique.

\title{
Conflicts of Interest
}

There is no conflict of interest

\author{
Fawad Zaman \\ Sungchang Lee \\ Mohamad K. A. Rahim \\ Sarmadullah Khan
}

\section{References}

[1] F. Zaman, "Joint angle-amplitude estimation for multiple signals with L-structured arrays using bioinspired computing," Wireless Communications and Mobile Computing, vol. 2017, Article ID 9428196, 12 pages, 2017.

[2] Y. Xiao, J. Xie, L. Huang, and H. C. So, "Multiantenna assisted source detection in toeplitz noise covariance," IEEE Signal Processing Letters, vol. 26, no. 6, pp. 813-817, 2019.

[3] S. Akbar, F. Zaman, M. Asif, A. U. Rehman, and M. A. Raja, "Novel application of FO-DPSO for 2-D parameter estimation of electromagnetic plane waves," Neural Computing and Applications, pp. 1-10, 2018.

[4] K. Luo and A. Manikas, "Joint transmitter-receiver optimization in multitarget mimo radar," IEEE Transactions on Signal Processing, vol. 65, no. 23, pp. 6292-6302, 2017.

[5] H. Guo, Z. Yang, Y. Zou et al., "Secure beamforming for cooperative wireless-powered networks with partial CSI," IEEE Internet of Things Journal, 2019. 


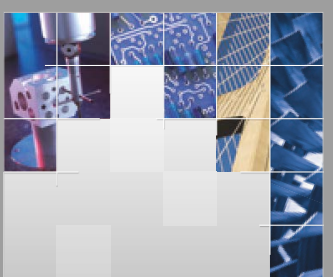

\section{Enfincering}
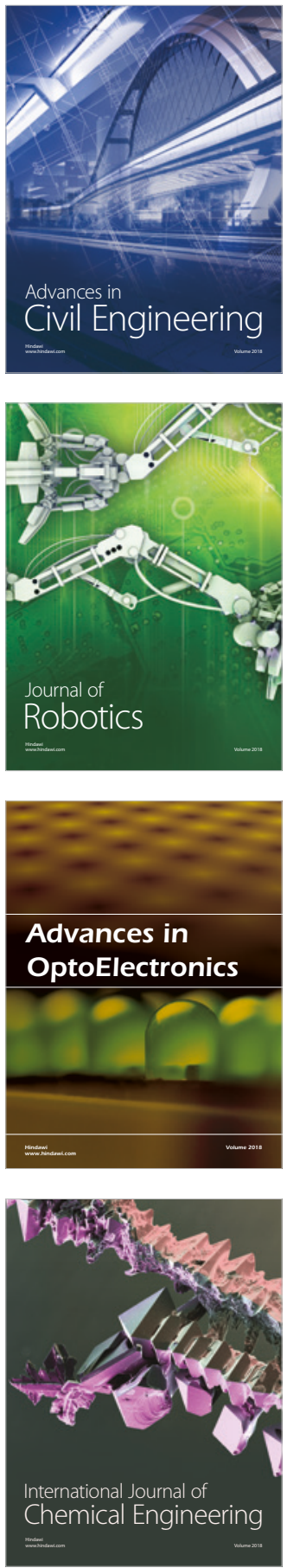

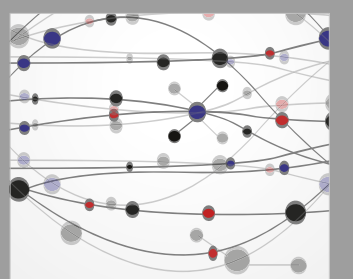

\section{Rotating \\ Machinery}

The Scientific World Journal

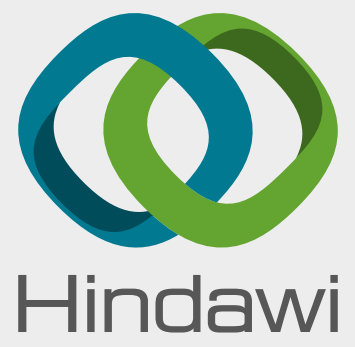

Submit your manuscripts at

www.hindawi.com
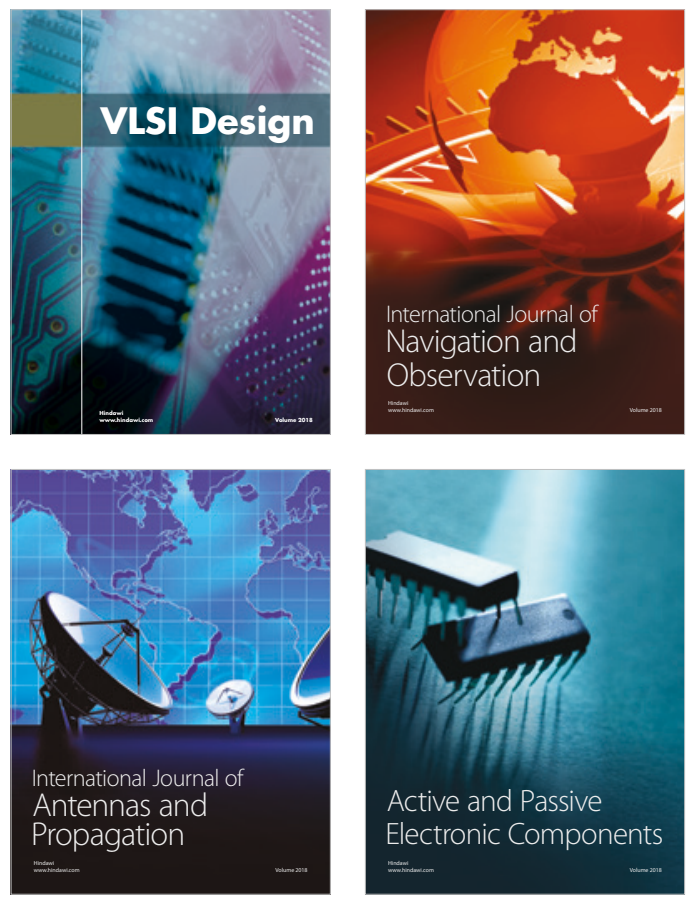
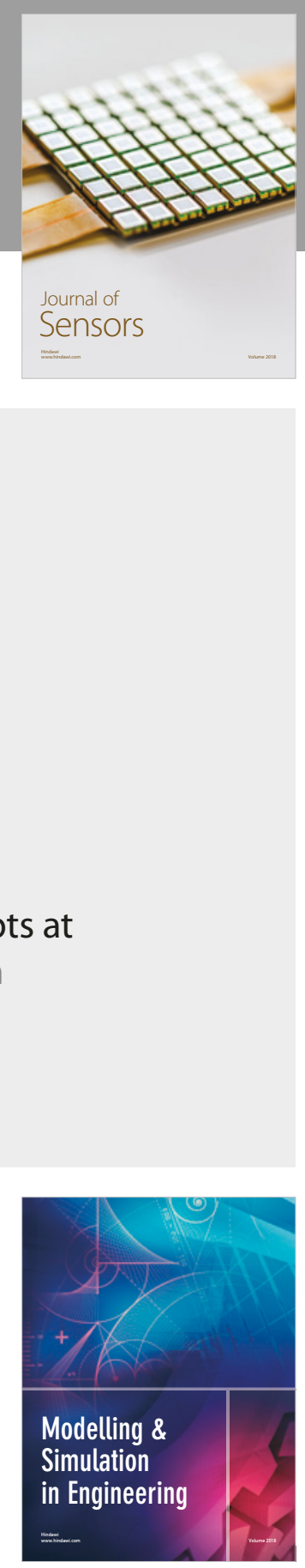

\section{Advances \\ Multimedia}
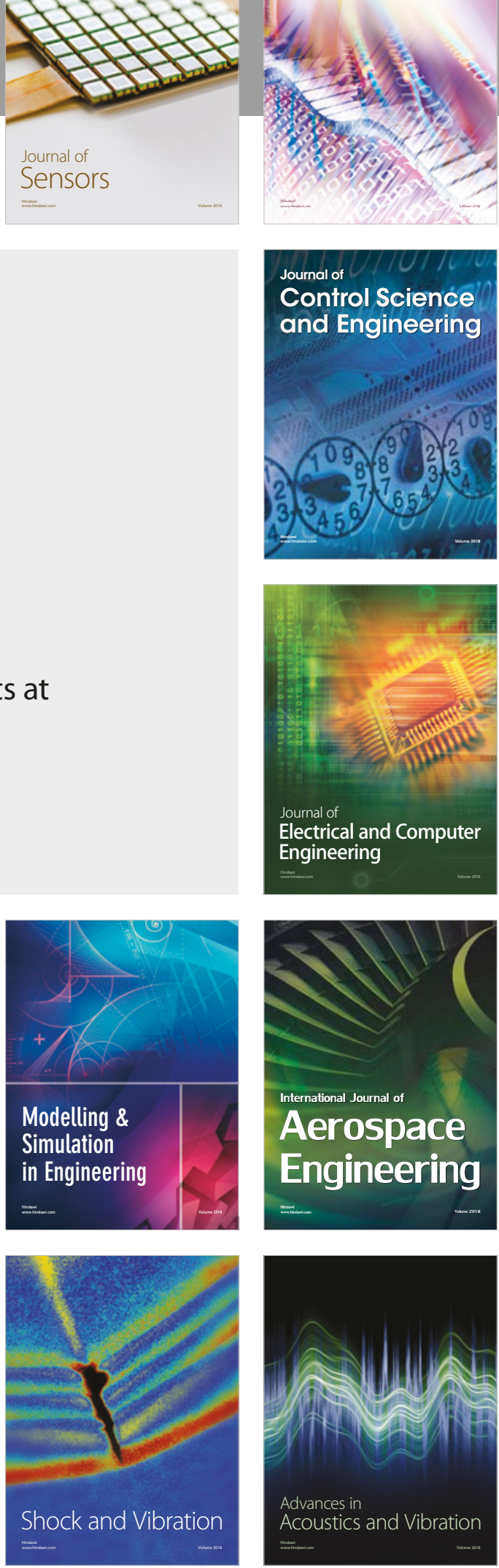